\title{
Long-Term Effects of Safinamide on Mood Fluctuations in Parkinson's Disease
}

\author{
Carlo Cattaneo $^{\mathrm{a}, *}$, Thomas Müller ${ }^{\mathrm{b}}$, Erminio Bonizzoni ${ }^{\mathrm{c}}$, Gabriele Lazzeri ${ }^{\mathrm{a}}$, Ioannis Kottakis ${ }^{\mathrm{a}}$ \\ and Charlotte Keywood ${ }^{\mathrm{d}}$ \\ ${ }^{a}$ Department of Medical, Zambon SpA, Bresso (MI), Italy \\ ${ }^{\mathrm{b}}$ Department of Neurology, St. Joseph Hospital Berlin-Weißensee, Berlin, Germany \\ ${ }^{\mathrm{c}}$ Department of Clinical Science and Community, Section of Medical Statistics and Biometry \\ "GA Maccacaro", University of Milan, Milan, Italy \\ ${ }^{\mathrm{d}}$ Department of R\&D, Zambon SpA, Bresso (MI), Italy
}

Accepted 23 July 2017

\begin{abstract}
.
Background: Mood disorders are very frequent in Parkinson's Disease (PD), and their effective treatment is still a major unresolved issue: growing evidence suggests that glutamatergic system dysfunction is directly involved. Safinamide is a drug with an innovative mechanism of action, dopaminergic and non-dopaminergic, that includes the reversible inhibition of the monoamine oxidase-B (MAO-B) enzyme and the modulation of excessive glutamate release through the use- and state-dependent blockade of the sodium channels.

Objective: To investigate the effects of safinamide on mood over two-year treatment in PD patients with motor fluctuations. Methods: This was a post-hoc analysis of the data from studies 016 and 018 . The analysis focused on outcomes related to mood, namely: scores of the "Emotional well-being" domain of the Parkinson's Disease Questionnaire (PDQ-39), scores of the GRID Hamilton Rating Scale for Depression (GRID-HAMD) and the proportion of patients reporting depression as an adverse event over the entire treatment period.

Results: Safinamide, compared to placebo, significantly improved the PDQ-39 "Emotional well-being" domain after 6-months $(p=0.0067)$ and 2 years $(p=0.0006)$, as well as the GRID-HAMD $(p=0.0408$ after 6 months and $p=0.0027$ after 2 years). Significantly fewer patients in the safinamide group, compared to placebo, experienced depression as adverse event ( $p=0.0444$ after 6 months and $p=0.0057$ after 2 years).

Conclusion: The favorable effect of safinamide on mood may be explained by the improvement in wearing off and by its modulation of glutamatergic hyperactivity and reversible MAO-B inhibition. Prospective studies are warranted to investigate this potential benefit.
\end{abstract}

Keywords: Glutamate, mood, Parkinson's disease, safinamide

\section{INTRODUCTION}

Parkinson's Disease (PD) is primarily considered as a motor disorder, but there is an increasing

\footnotetext{
*Correspondence to: Dr. Carlo Cattaneo, DBSc CAAN, Corporate Medical Advisor CNS and Neurological Rare Diseases, Zambon SpA, Via Lillo del Duca 10, 20091 Bresso (Milan), Italy. Tel.: +39 02 66524299; Mobile: +39 347 6174245; Fax: +39 02 66524887; E-mail: carlo.cattaneo@zambongroup.com.
}

recognition of a wide range on non-motor symptoms (NMS) such as low mood, pain, fatigue and sleep disorders, which often occur before motor symptoms [1].

Complex, interconnected neuronal pathways, regulated by a number of different neurotransmitters in addition to dopamine, are involved in the etiology of motor and non-motor symptoms. The development of agents interacting with several neurotransmission 
systems in the CNS might be very useful for the treatment of this disease [2].

Between NMS, mood disorders have been reported in up to $35 \%$ of PD patients, with a substantial negative impact on the quality of life of patients and their caregivers. These symptoms are often underdiagnosed and therefore undertreated; however, to optimize patients outcomes, they need to be managed effectively [3].

Glutamate is the most abundant excitatory neurotransmitter in the mammalian brain, and modulates many physiological brain functions, with a crucial role in synaptic transmission and plasticity. Several clinical studies have supported a key role for the glutamatergic system in the pathophysiology of mood disorders [4].

Drugs that inhibits voltage-activated channel activity (e.g. riluzole or lamotrigine), decreasing abnormal presynaptic glutamate release, are considered mood stabilizers [5].

Safinamide has a dual mechanism of action, dopaminergic and non-dopaminergic, that offers a unique approach to the management of motor symptoms and motor complications. The nondopaminergic mechanism of action might be useful for the treatment of non-motor symptoms: safinamide, in fact, suppresses repetitive firing by useand state-dependent sodium channel blockade, thus reducing excessive release of glutamate [6], and has shown to improve PD-related chronic pain [7]. This post-hoc analysis of the data from trials 016 and 018 was performed to evaluate the effects of safinamide $100 \mathrm{mg} /$ day on mood disorders in PD patients with motor fluctuations.

\section{MATERIALS AND METHODS}

\section{Patients}

Study 016 (NCT01187966) was a Phase III, 24-week, double blind, placebo-controlled, parallelgroup, randomized, multicentre and multinational trial comparing safinamide versus placebo as addon therapy to stable doses of levodopa (alone or with other antiparkinson drugs) in patients with mid- to late-stage PD and motor fluctuations. The primary efficacy endpoint was the change from baseline to week 24 in daily $\mathrm{ON}$ time with no/non-troublesome dyskinesia as recorded by patients' diaries and the main secondary efficacy endpoint was the reduction in OFF time [8].
Study 018 (NCT01286935) was the long-term extension (up to 24 months) of study 016. The primary endpoint was the mean change from baseline (study 016 start) to study end (two years) in the total Dyskinesia Rating Scale (DRS) score evaluated during $\mathrm{ON}$ time. The main secondary efficacy endpoints were changes in ON time (without troublesome dyskinesia) and OFF time [9].

Both protocols and patient materials were approved by Independent Ethics Committees and Health Authorities in all the participating countries. All patients signed an informed consent form and the studies were conducted according to the Declaration of Helsinki.

Patients with depressive symptoms and those already treated with antidepressant were excluded. Treatments with tri-tetracyclic antidepressants and serotonin-norepinephrine reuptake inhibitors (SNRIs) were not permitted in both studies. Selective serotonin reuptake inhibitors (SSRIs) were allowed at study entry at the lowest therapeutic dose and had to remain stable throughout the trials. The doses of levodopa and other PD treatments were optimized before study start and should remain stable, whenever possible, during the treatment period. However, in both studies the doses could be changed in case of deterioration in motor symptoms or occurrence of adverse events.

Safinamide significantly improved the daily ON time (with no/non-troublesome dyskinesia) and reduced the OFF time maintaining the efficacy up to two years. In the trial 018 , the primary endpoint (reduction in dyskinesia) was not met, despite a substantial decrease in DRS score in the safinamide group compared to placebo. Nonetheless, in the subgroup of patients with moderate-severe dyskinesia at baseline, safinamide $100 \mathrm{mg} /$ day significantly improved the Dyskinesia Rating Scale score. The incidence of treatment-emergent adverse events and serious adverse events was similar in safinamide and placebo groups.

\section{Outcome measures}

This is a post-hoc analysis of the data from studies 016 and 018 , evaluating the effects of safinamide $100 \mathrm{mg} /$ day oral tablets versus placebo on the scores of the PDQ-39 "Emotional well-being" domain, the scores of the GRID-HAMD scale and on the proportion of patients reporting the adverse event "depression". 


\section{Statistical methods}

Comparisons of the mean change from baseline (PDQ-39, GRID-HAMD) for the active-treatment group to placebo were performed using ANCOVA models with treatment group and centre as fixed dummy effects and baseline value as continuous covariate. Results are reported as least square means with associated two-tailed $95 \%$ confidence intervals (CIs) and two-tailed $P$-values.

For dichotomous outcome variables (depression adverse events), the absolute number and proportion in percent were tabulated together with two-sided $P$-values for the active-treatment group to placebo which were computed using the Fisher's Exact test.

The intention-to-treat (ITT) patient populations were used for all post hoc analyses while the last observation carried forward (LOCF) approach was applied to account for missing data at study termination. SAS software version 9.4 was used for all statistical analyses.

\section{RESULTS}

Results of the analyses of the changes from baseline to 6 and 24 months in the PDQ-39 "Emotional well-being" domain in studies 016 and 018 are shown in Fig. 1.

In study 016 the LS mean change from baseline was -5.14 (95\% CI: $-7.98,-3.54)$ for the safinamide group and -1.37 (95\% CI: $-3.65,+0.31)$ for the placebo group.

In study 018 the LS mean change from baseline was -4.56 (95\% CI: $-7.40,-3.46)$ for the safinamide group and $+0.10(95 \% \mathrm{CI}:-0.07,+0.97)$ for the placebo group.

Safinamide $100 \mathrm{mg} /$ day was associated with significantly greater improvements from baseline to 6 months of the score of the PDQ-39 domain "Emotional well-being" compared with placebo: mean difference vs placebo for the changes from baseline -3.77 (95\% CI: $-6.49,-1.05 ; p=0.0067)$. The improvements observed were maintained in the longterm: mean difference of safinamide vs placebo -4.66 (95\% CI $-7.30,-2.02 ; p=0.0006$ ). Results of the analyses of the changes from baseline to 6 and 24 months in the GRID-HAMD scores in studies 016 and 018 are shown in Fig. 2.

In study 016 the LS mean change from baseline was -1.06 (95\% CI: $-1.57,-0.67)$ for the safinamide group and -0.49 (95\% CI: $-0.97,-0.07$ ) for the placebo group. In study 018 the LS mean change from baseline was -0.76 (95\% CI: $-1.40,-0.59)$ for the safinamide group and +0.11 (95\% CI: $-0.14,+0.95$ ) for the placebo group.

Safinamide $100 \mathrm{mg} /$ day resulted also in greater improvements in GRID-HAMD scores from baseline, compared with placebo (mean difference vs placebo $-0.57 ; 95 \% \mathrm{CI}-1.13,-0.02 ; p=0.0408)$ that were maintained in the long-term (mean difference of

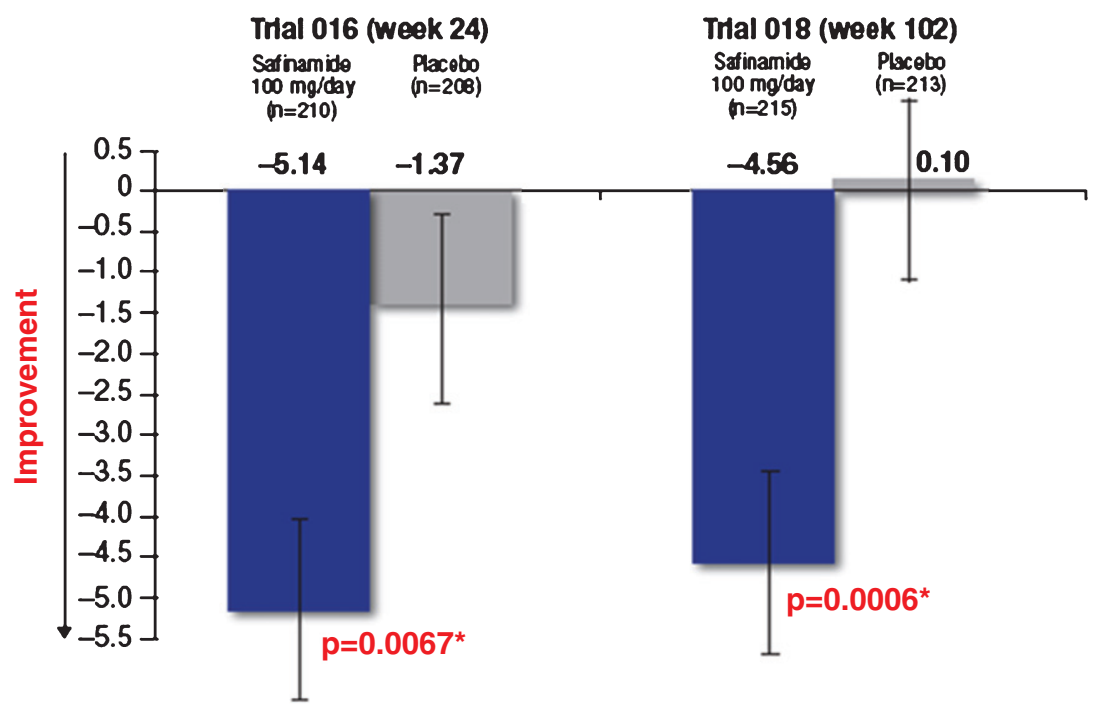

two-sided t-test

Fig. 1. Trials 016 and 018: changes (LS means \pm SD) in PDQ-39 "Emotional well-being" scores. LS = least squares; SD = standard deviation; PDQ-39= Parkinson's Disease Questionnaire 39 items. 


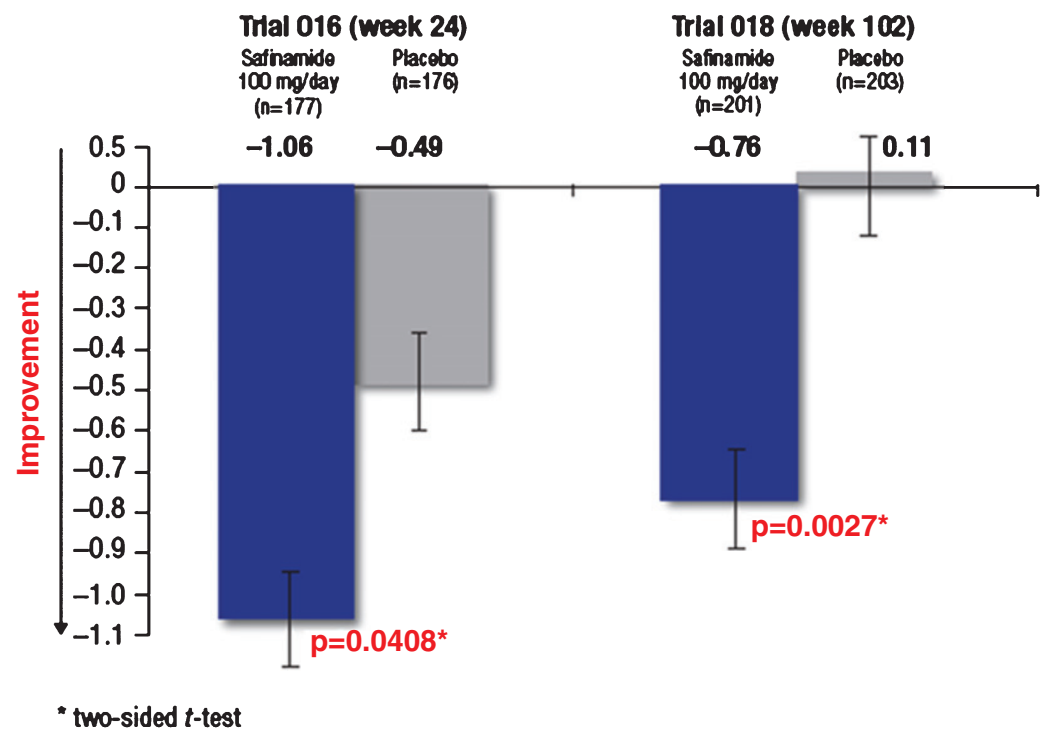

Fig. 2. Trials 016 and 018: changes (LS means \pm SD) in GRID-HAMD scores. LS=least squares; SD=standard deviation; GRIDHAMD $=$ Grid version of the Hamilton Rating Scale for Depression.

Table 1

Trials 016 and 018: proportion of patients reporting depression as adverse event

\begin{tabular}{|c|c|c|c|c|c|c|}
\hline \multirow[t]{2}{*}{ Trial } & \multirow[t]{2}{*}{ Visit } & \multicolumn{2}{|c|}{$\begin{array}{l}\text { Safinamide } \\
100 \mathrm{mg} / \text { day }\end{array}$} & \multicolumn{2}{|c|}{ Placebo } & \multirow[t]{2}{*}{$P$-value ${ }^{\dagger}$} \\
\hline & & $\mathrm{N}$ & $\mathrm{n}(\%)$ & $\mathrm{N}$ & $\mathrm{n}(\%)$ & \\
\hline 016 & week 24 & 224 & $4(1.8 \%)$ & 222 & $12(5.4 \%)$ & 0.0444 \\
\hline 018 & week 102 & 180 & $3(1.7 \%)$ & 175 & $14(8.0 \%)$ & 0.0057 \\
\hline
\end{tabular}

${ }^{\dagger}$ Two-sided Fisher's Exact test. $\mathrm{N}=$ total number of patients; $\mathrm{n}(\%)=$ number (percentage) of patients reporting depression as adverse event.

safinamide vs placebo $-0.87 ; 95 \%$ CI $-1.44,-0.30$; $p=0.0027$ ).

After 6 months, significantly fewer patients receiving safinamide $100 \mathrm{mg}$ /day reported depression as adverse event compared with those receiving placebo (respectively, $1.8 \%$ vs $5.4 \%, p=0.0444$ ). The proportion of patients with depression as adverse event remained stable in the safinamide group over the extension period, while increased in the placebo group (at 24 months, respectively, $1.7 \%$ vs $8.0 \%$, $p=0.0057$ ) (Table 1).

\section{DISCUSSION}

PD is a complex neurodegenerative illness with both motor and non-motor symptoms. During the course of the disease, many patients experience some mood disturbances including depression and anxiety, characterized by major disruption in their mood and emotions [10]. This affective symptomatology is costly from economic, social and personal standpoints.

Even if mood deteriorations can arise as a result of specific parkinsonian symptoms, such as motor fluctuations [11], dysfunction in dopaminergic pathways contributes to mood disorders. Pramipexole, a dopamine-agonist, has been shown to improve depressive symptoms in PD patients [12], and inhibition of monoamine oxidase enzyme increases the levels of biogenic amines (in particular dopamine) in the synaptic cleft, enhancing mood and motivation [13].

Moreover, recent studies have provided strong evidence that glutamate and other neurotransmitters, in addition to monoamines, are involved in the pathophysiology and treatment of mood disorders [14]. Glutamate levels are tightly regulated by sodiumdependent glutamate transporters, which maintain low concentration of the neurotransmitter protecting neuronal cells against excitotoxicity [15].

Blockade of voltage-dependent sodium channels is functionally related to the inhibition of excessive glutamate. Mood stabilizing agents, like valproic acid, lamotrigine and riluzole, modulate glutamate neurotransmission by blocking sodium channels [16].

The add-on of safinamide $100 \mathrm{mg} /$ day to a stable dose of levodopa (alone or in combination with other PD medications) was associated with an improve- 
ment in mood in PD patients over two years. During the entire treatment period, significantly fewer patients receiving safinamide experienced depression as adverse event compared with patients receiving placebo. These results could certainly be a consequence of the improvement in motor symptoms and fluctuations, but this may not be the only explanation. After 2-years treatment, there was an increase in ON time and a decrease in OFF time in both placebo and safinamide groups, albeit to a greater extent in the safinamide group [9]. However, the positive effect of treatment on mood scores was substantially greater in the safinamide group than the placebo group suggesting that the benefit may not be solely due to improved motor symptom control. The mechanism of glutamatergic modulation cannot be ruled out as contributing to the positive effect seen on mood in the safinamide group.

The notion that the beneficial effect of safinamide may be explained by both its reversible MAO-B inhibition and by the modulation of glutamatergic hyperactivity, is supported by the finding that rasagiline, which is solely a MAO-B inhibitor, has been found to have no significant effects on depression in PD patients [17].

There are some limitations to be considered in this post-hoc analysis: the original trials were not designed to investigate mood as a primary endpoint, and patients with depressive symptoms and those already treated with antidepressant were excluded. These findings should therefore be considered as exploratory, and must be confirmed in larger clinical trials.

\section{Conclusion}

Mood disorders have been reported as a frequent comorbidity in PD and can occur in both early and late stages of the pathology. In some cases, they may precede the development of motor fluctuations affecting the quality of life and the daily function of patients [18].

While the treatment paradigms for motor symptoms are well established, there is a lack of clear guidelines pertaining to the cure of the emotional disturbances.

Despite some limitations, the results of these analyses showed that safinamide $100 \mathrm{mg}$ /day may have a favorable effect on mood that might be explained by the improvement in wearing off and by its modulation of glutamatergic hyperactivity and reversible MAO-B inhibition.
Further investigations are necessary to assess the potential benefits of safinamide on mood and their clinical relevance.

\section{CONFLICT OF INTEREST}

Thomas Müller is member of the Scientific Advisory Board of Zambon SpA. Carlo Cattaneo, Gabriele Lazzeri, Charlotte Keywood, and Ioannis Kottakis are employees at Zambon SpA, the Pharmaceutical Company that is marketing safinamide in Europe. Erminio Bonizzoni is a consultant statistician for Zambon SpA.

\section{REFERENCES}

[1] Korczyn AM, Chauduri RK, \& Van Laar T (2015) Extending our understanding of the dopaminergic basis of non-motor symptoms in Parkinson's Disease. Eur Neurol Review, 10, 23-27.

[2] Fox SH (2013) Non-dopaminergic treatments for motor control in Parkinson's disease. Drugs, 73, 1405-1415.

[3] Leentjens AF, Koester J, Fruh B, Shephard DT, Barone P, \& Houben JJ (2009) The effect of pramipexole on mood and motivational symptoms in Parkinson's disease: A metaanalysis of placebo-controlled studies. Clin Ther, 31, 89-98.

[4] Machado-Vieira R, Manji HK, \& Zarate CA (2009) The role of the tripartite glutamatergic synapse in the pathophysiology and therapeutics of mood disorders. Neuroscientist, 15, 525-539.

[5] Zarate C, Machado-Vieira R, Henter I, Ibrahim L, Diazgranados N, \& Salvatore G (2010) Glutamatergic modulators: The future of treating mood disorders? Harv Rev Psychiatry, 18, 293-303.

[6] Caccia C, Maj R, Calabresi M, Maestroni S, Faravelli L, Curatolo L, Salvati P, \& Fariello RG (2006) Safinamide: From molecular targets to a new anti-Parkinson drug. $\mathrm{Neu}$ rology, 67, S18-S23.

[7] Cattaneo C, Barone P, Bonizzoni E, \& Sardina M (2017) Effects of safinamide on pain in fluctuating Parkinson's Disease patients: A post-hoc analysis. J Parkinsons Dis, 7, 95-101.

[8] Borgohain R, Szasz J, Stanzione P, Meshram C, Bhatt M, Chirilineau D, Stocchi F, Lucini V, Giuliani R, Forrest E, Rice P, \& Anand R (2014) Randomized trial of safinamide add-on to levodopa in Parkinson's disease with motor fluctuations. Mov Disord, 29, 229-237.

[9] Borgohain R, Szasz J, Stanzione P, Chandrashekar M, Bhatt M, Chirilineau D, Stocchi F, Lucini V, Giuliani R, Forrest E, Rice P, \& Anand R (2014) Two-year, randomized, controlled study of safinamide as add-on to levodopa in mid to late Parkinson's Disease. Mov Disord, 29, 1273-1280.

[10] Chaudhuri KR, Healy DG, \& Shapira AH (2006) Non-motor symptoms of Parkinson's Disease: Diagnosis and management. Lancet Neurol, 5, 235-245.

[11] Menza M, Sage J, Marshall E, Cody R, \& Duvoisin R (1990) Mood changes and "On-Off" phenomena in Parkinson's Disease. Mov Disord 5, 148-151.

[12] Barone P, Poewe W, Albrecht S, Debieuvre C, Massey D, Rascol O, Tolosa E, \& Weintraub D (2010) Pramipexole for the treatment of depressive symptoms in patients with 
Parkinson's disease: A randomised, double-bind, placebocontrolled trial. Lancet Neurol, 22, 573-580.

[13] Riederer P, \& Müller T (2017) Use of monoamine oxidase inhibitors in chronic neurodegeneration. Expert Opin Drug Metab Toxicol, 13, 233-240.

[14] Kugaya A, \& Sanacora G (2005) Beyond monoamines glutamatergic function in mood disorders. CNS Spectr, 10, 808-819.

[15] Salvati P, Maj R, Caccia C, Cervini MA, Fornaretto MG, Lamberti E, Pevarello P, Skeen GA, White HS, Wolf HH, Faravelli L, Mazzanti M, Mancinelli E, Varasi M, \& Fariello RG (1999) Biochemical and electrophysiological studies on the mechanism of action of PNU-151774E, a novel antiepileptic compound. J Pharmacol Exp Ther, 288, 1151-1159.

[16] Miladinovic T, Nashed MG, \& Singh G (2015) Overview of glutamatergic dysregulation in central pathologies. Biomolecules, 5, 3122-3141.

[17] Barone P, Santangelo G, Morgante L, Onofrj M, Meco G, Abruzzese G, Bonuccelli U, Cossu G, Pezzoli G, Stanzione P, Lopiano L, Antonini A, \& Tinazzi M (2015) A randomized clinical trial to evaluate the effects of rasagiline on depressive symptoms in non-demented Parkinson's disease patients. Eur J Neurol, 22, 1184-1191.

[18] Tan LC (2012) Mood disorders in Parkinson's disease. Parkinsonism Relat Disord, 18(Suppl 1), S74-S76. 